\title{
Obesity in COVID-19: A Systematic Review and Meta-analysis
}

\author{
Jamie SY $\underline{\text { Ho}},{ }^{{ }^{1}}{ }_{M B B C h i r}$, Daniel I Fernando, ${ }^{* 1}$ MBBChir, Mark Y $\underline{\text { Chan }},{ }^{2,3}{ }_{M B B S}$, Ching-Hui Sia,,${ }^{2,3}$ MBBS
}

\begin{abstract}
Objective: Obesity has been shown to be associated with adverse outcomes in viral infections such as influenza, but previous studies on coronavirus disease 2019 (COVID-19) had mixed results. The aim of this systematic review is to investigate the relationship between COVID-19 and obesity.

Methods: We performed a systematic review and meta-analysis. A literature search of MEDLINE, EMBASE, Scopus, Web of Science, CENTRAL, OpenGrey and preprint servers medRxiv and bioRxiv was performed, with no restriction on language or date of publication. Primary outcomes of this study were intensive care unit (ICU) admission or critical disease, severe disease and mortality. Secondary outcome was a positive COVID-19 test. Meta-analysis was performed using OpenMeta-Analyst software, and heterogeneity was tested using Cochran's Q test and $I^{2}$ statistic. The study protocol was registered on PROSPERO (CRD42020184953).

Results: A total of 1,493 articles were identified and 61 studies on 270,241 patients were included. The pooled prevalence of obesity was $27.6 \%$ (95\% confidence interval [CI] 22.0-33.2) in hospitalised patients. Obesity was not significantly associated with increased ICU admission or critical illness (odds ratio [OR] 1.25, 95\% CI 0.99-1.58, $P=0.062, I^{2}=31.0$ ) but was significantly associated with more severe disease (OR 3.13, 95\% CI 1.41-6.92, $P=0.005, P=82.6$ ), mortality (OR 1.36, 95\% CI $1.09-1.69, P=0.006$, $\left.I^{2}=88.5\right)$ and a positive COVID-19 test (OR $1.50,95 \%$ CI $\left.1.25-1.81, P<0.001\right)$.

Conclusion: Obesity increased the risk of severe disease, mortality and infection with COVID-19. Higher body mass index was associated with ICU admission and critical disease. Patients who are obese may be more susceptible to severe acute respiratory syndrome coronavirus 2 (SARS-CoV-2) infection, and infected patients should be monitored closely for adverse outcomes.
\end{abstract}

Ann Acad Med Singap 2020;49:996-1008

Keywords: Body mass index, coronavirus, intensive care, mortality, prognosis

\section{Introduction}

Coronavirus disease 2019 (COVID-19) is an ongoing global pandemic infection by the severe acute respiratory syndrome coronavirus 2 (SARS-CoV-2). It is established that increasing age and comorbidities such as cardiovascular diseases are associated with risk of infection, more severe disease and adverse outcomes. ${ }^{1}$
Obesity is an epidemic globally, causing more than 2.8 million deaths per year worldwide in 2019. The World Health Organization (WHO) defines obesity as a body mass index (BMI) of $\geq 30$, and overweight as a BMI of $\geq 25$. Other definitions of obesity include the WHO Asia-Pacific BMI guidelines, which defines obese as $\mathrm{BMI} \geq 27.5$ and overweight as $\mathrm{BMI} \geq 23$. ${ }^{2}$

\footnotetext{
${ }^{1}$ School of Clinical Medicine, University of Cambridge, Cambridge, UK

${ }^{2}$ Department of Cardiology, National University Heart Centre, Singapore

${ }^{3}$ Department of Medicine, Yong Loo Lin School of Medicine, National University of Singapore, Singapore

Address for Correspondence: Dr Ching-Hui Sia, Department of Cardiology, National University Heart Centre, 5 Lower Kent Ridge Rd, Singapore 119074.

Email: ching_hui_sia@nuhs.edu.sg

*Joint first authors
} 
Obese patients were at increased risk of hospitalisation, complications and death in the 2009 influenza A (H1N1) pandemic. ${ }^{3}$ There are suggestions that, similar to influenza, obesity may be a significant risk factor in COVID-19. Possible pathophysiology includes a chronic proinflammatory environment, reducing immunological response to infections, and altered dynamics of pulmonary ventilation with reduced diaphragmatic expansion and increased anatomical dead space. ${ }^{4}$ However, the evidence from reported cohort studies and case series has been mixed. ${ }^{5,6}$ In this comprehensive systematic review and meta-analysis of the current literature on obesity and COVID-19, we aimed to characterise the relationship between obesity and adverse prognostic outcomes.

\section{Methods}

The protocol for this review was registered and published on the International Prospective Register of Systematic Reviews (PROSPERO; CRD42020184953). A literature search of MEDLINE, EMBASE, Scopus, Web of Science, CENTRAL, OpenGrey and preprint servers medRxiv and bioRxiv was performed on 8 May 2020, using the search terms "coronavirus or COVID" or "SARS-COV-2" or similar terms to the pandemic, and "obesity or hyperphagia or overweight" or words to that effect. Additional articles were identified from hand searching of the reference lists of included studies. Inclusion criteria were clinical studies (1) that reported obesity prevalence or outcomes, and (2) that were performed on COVID-19 patients. All research study types, such as case series, cohort studies, longitudinal studies and randomised controlled trials, were included, with no restriction on publication date or language of publication. Articles discussing other infectious outbreaks and studies on animals or in vitro studies were excluded. Other systematic reviews, literature reviews, editorials and opinion articles were excluded, but references were screened for relevant articles. Articles from the Chinese WanFang and SinoMed databases were not searched owing to language limitations.

Titles and abstracts were screened independently by 2 researchers, and discrepancies were resolved through discussion or involvement of a third researcher. Full texts were identified, and data were extracted onto a standardised data extraction form by the 2 independent researchers. The extracted data included study type, patient characteristics, prevalence of obesity, and clinical outcomes such as severe disease, intensive care unit (ICU) admission and mortality. The quality of the included studies was assessed using the Newcastle-
Ottawa Scale for case series, cohort, cross-sectional and case-control studies.

The primary outcomes of this systematic review were prevalence of obesity in COVID-19 patients, and association of obesity with adverse outcomes such as ICU admission, critical illness, severe disease and mortality. The secondary outcome was a positive COVID-19 test.

\section{Definitions}

The definitions of terms including "obesity", "severe disease" and "critical disease" used for each individual study were adopted in this meta-analysis. Both European and Asian definitions of obesity were used, for example, based on the geographical origin of the study. Critical illness was defined as any of the following: respiratory failure requiring invasive mechanical ventilation, shock, or any other organ failure, requiring intensive therapy unit (ITU) or ICU monitoring and treatment. Severe disease was defined according to the WHO interim guidance, and its definition was used by most study types that made the distinction between different severities of COVID-19.

For "positive COVID-19 test" results to be included in the analyses, COVID-19 needs to be diagnosed by reverse transcription-polymerase chain reaction (RT-PCR) testing using a nasopharyngeal swab.

\section{Statistical analyses}

A meta-analysis was performed for the primary and secondary outcomes using the OpenMeta-Analyst software. DerSimonian and Laird's random effects model was employed to calculate the pooled odds ratio (OR) and 95\% confidence interval (CI). Study heterogeneity was assessed using Cochran's Q test and $I^{2}$ statistic. A $P$ value of $<0.05$ was considered statistically significant.

\section{Results}

In total, 61 studies on 270,241 patients fit the inclusion criteria, including 38 cohort studies, 2 case-control studies, 8 cross-sectional studies and 13 case series (Fig. 1). The characteristics of the included studies are presented in Table 1 . The quality of studies was generally moderate to good, with the exception of small case series that had a high risk of selection bias and lack of control group. A total of 46 studies were included in the metaanalysis, with any outcome analysed. The remaining 15 studies were descriptive studies including case series and case reports, and their reported patient characteristics are presented in Table 1. The prevalence of obesity was 


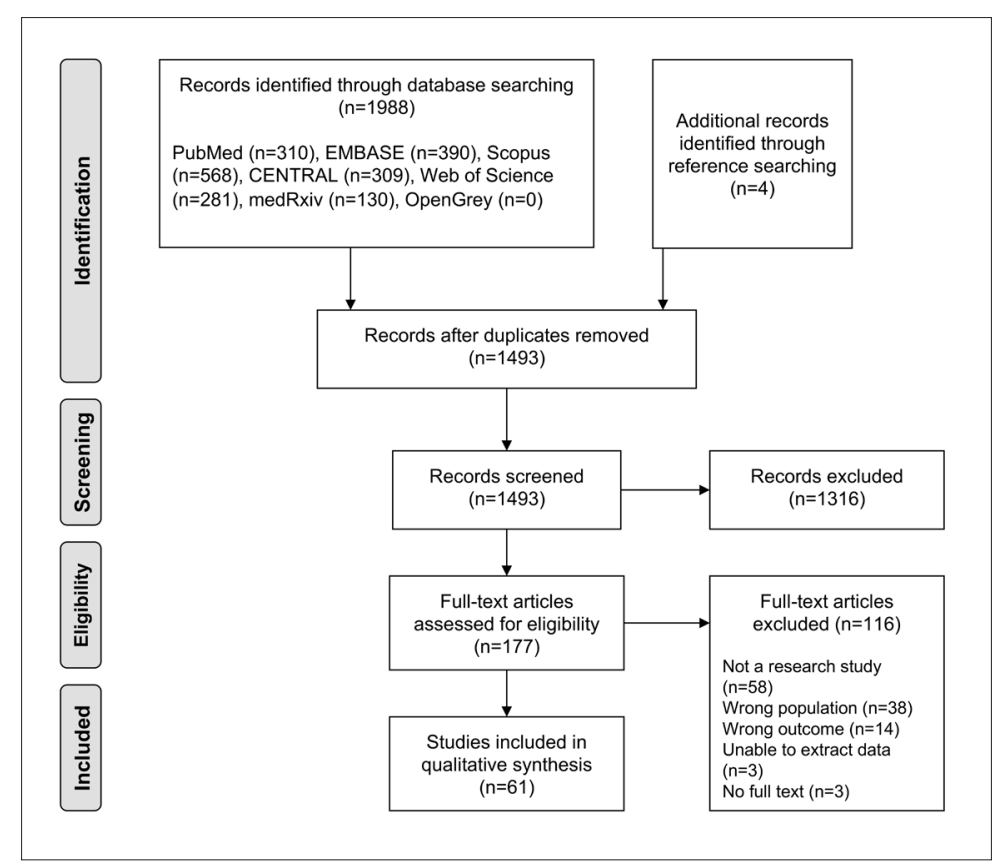

Fig. 1. Preferred Reporting Items for Systematic Reviews and Meta-Analyses (PRISMA) flow diagram of the process of literature search, screening and inclusion of studies

$27.6 \%(95 \%$ CI 22.0-33.2) across 31 cohort studies in the hospital setting, ${ }^{7-35}$ and univariate meta-regression model revealed no significant association with sample size (regression co-efficient $<0.0001, P=0.921$ ). Only 31 cohort studies reported prevalence of obesity in the hospital setting and so were included with the pooled prevalence meta-analysis. On exclusion of 4 Chinese studies owing to ethnic differences in BMI, ${ }^{14,26,27,31}$ the prevalence of obesity was $31.1 \%$ (95\% CI 25.1-37.2). The prevalence of obesity in Chinese studies alone was $5.4 \%$ (95\% CI $1.9-8.9 \%)$.

\section{ICU admission and critical illness}

A total of 12 studies reported the outcome of ICU admission or critical illness, and comprised 9 cohort studies (8 retrospective, 1 prospective), 2 case series and 1 cross-sectional study, involving 10,314 patients with COVID-19.7,11,12,14-16,28,36-40 All studies were of moderate to good quality and were conducted in the hospital setting. The proportion of obese patients (BMI $\geq 30$ ) in ICU or critical patients was compared with that of non-ICU hospitalised patients in 8 studies involving 9,869 patients. , $11,12,14-16,28,36$ On meta-analysis, obesity was not significantly associated with increased ICU admission or critical illness (OR 1.25, 95\% CI $0.99-1.58, P=0.062)$, with moderate heterogeneity $\left(\mathrm{Q}=10.1, I^{2}=31.0\right)$ (Fig. 2A).

Five studies involving 1,445 participants reported mean or median BMI of ICU patients and non-ICU patients in the hospital setting (Fig. 2B). ${ }^{7,37-40}$ The ICU or critical illness group had a mean BMI of 28.7 (26.1-31.4) while the non-ICU group had a BMI of 25.2 (95\% CI 22.0-28.5). The overall mean difference in BMI was $2.32(95 \%$ CI 1.04-3.60, $P<0.001)$, with substantial heterogeneity $\left(\mathrm{Q}=13.0, I^{2}=69.2\right)$. In particular, the study by Lau et al. showed a particularly large effect size and 95\% CI, and the study focused on ICU admissions with recorded levels of serum 25-hydroxycholecalciferol in a single centre, which may not be representative of typical patients admitted to ICU for COVID-19. ${ }^{37}$ There may also be ethnic differences across study populations, as the 2 studies from China ${ }^{39,40}$ reported lower BMIs than those from the US and Mexico, ${ }^{7,37,38}$ although the mean differences were found to be similar in magnitude.

Three studies found an increased risk of invasive mechanical ventilation in patients with obesity, ${ }^{18,21,34}$ including 1 study with a large cohort of 20,737 patients in the US. ${ }^{34}$ A cohort study from France ${ }^{5}$ found that the risk of intubation increased in $124 \mathrm{ICU}$ patients with $\mathrm{BMI} \geq 35$ (OR 7.36, 95\% CI 1.63-33.14) but not in patients with BMI 30-35 (OR 3.45, 95\% CI 0.83-14.31), which was similar to another cohort study involving 291 patients in France with BMI $\geq 35$ (OR 6.24, 95\% CI 2.30-16.93) and BMI 30-35 (OR 1.97, 95\% CI 1.00-3.90). A case series of 2 obese patients reported perforation of the membranous trachea or cricoid membrane during intubation for acute 


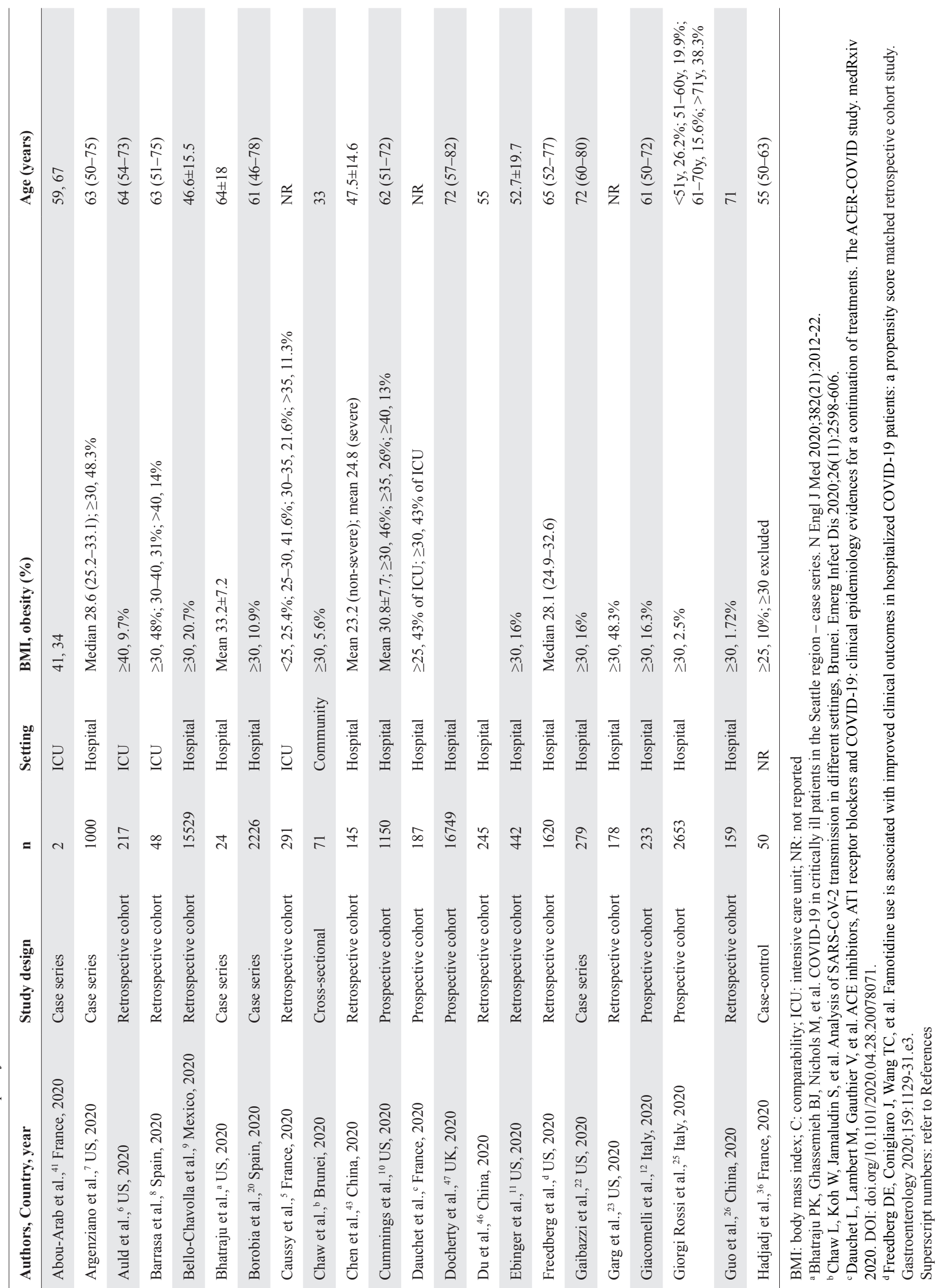




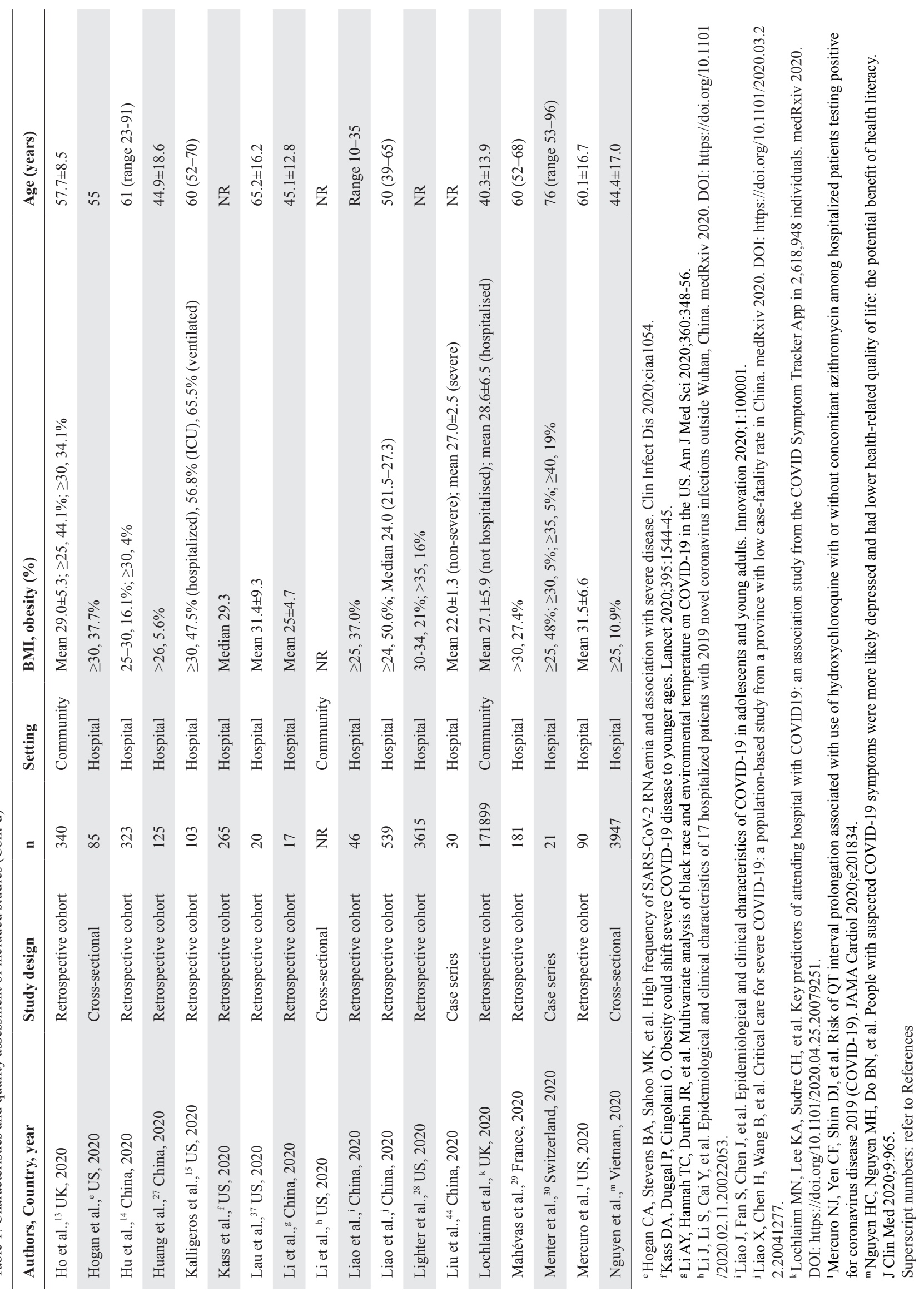




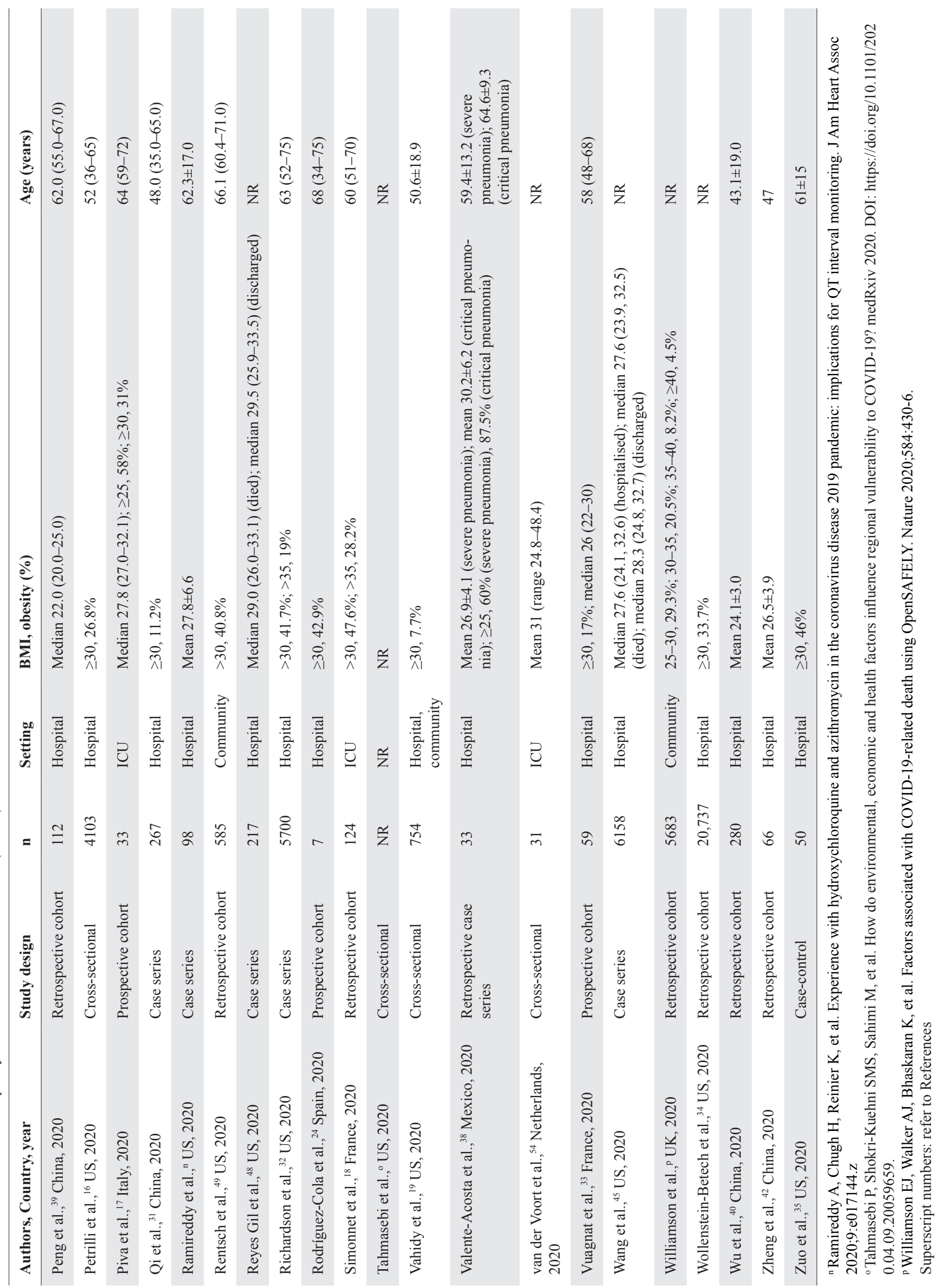




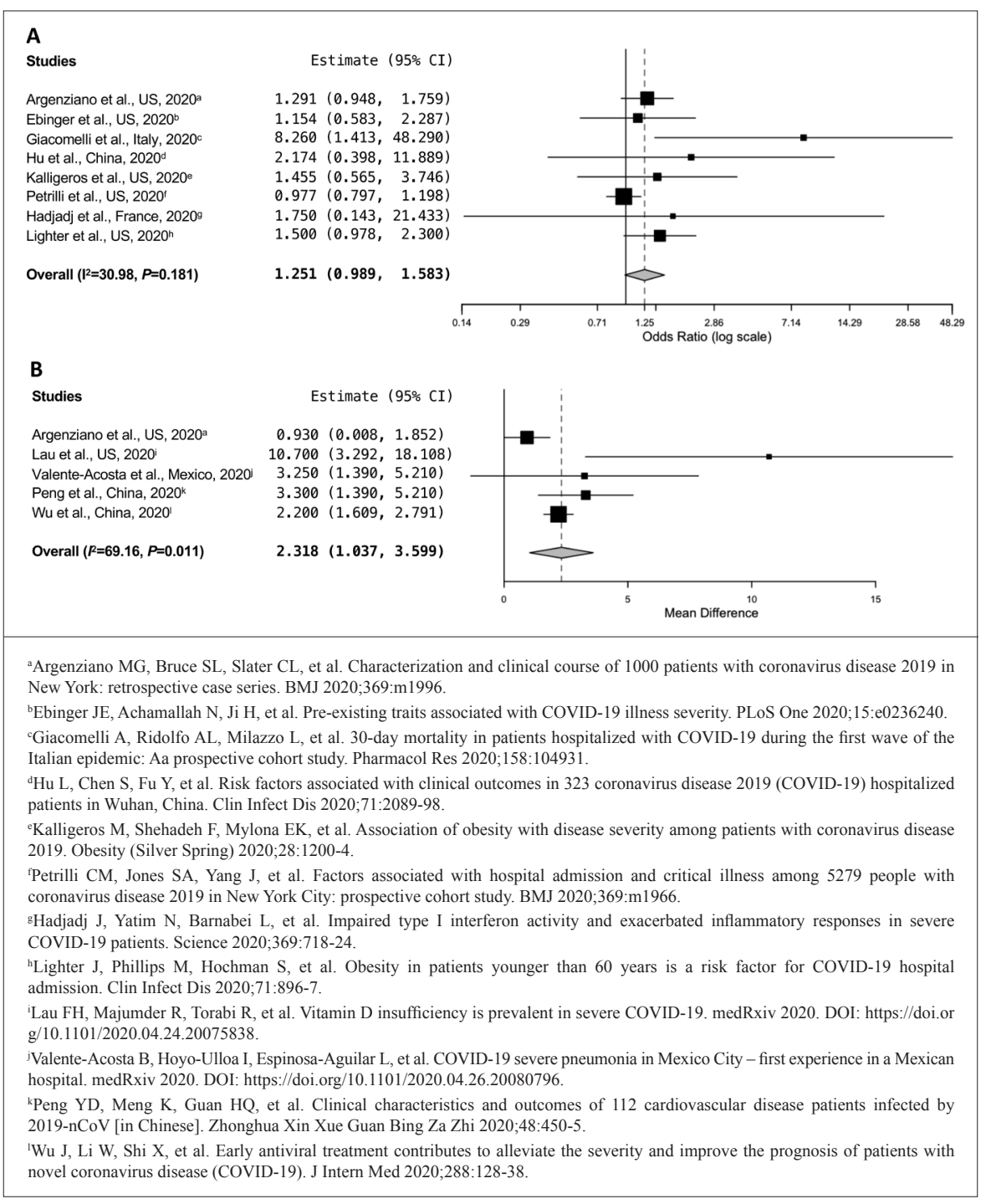

Fig. 2. Meta-analysis of the proportion of COVID-19 patients with obesity, comparing (A) the prevalence in intensive care unit (ICU) or with critical illness, to that in hospitalised non-ICU patients; and (B) the mean body mass index in ICU patients to that in non-ICU patients.

CI: confidence interval; $P$ : I-squared statistic of heterogeneity

respiratory distress syndrome, which was refractory to prone positioning and non-invasive ventilation. ${ }^{41}$

\section{Severe disease}

Severe disease was defined according to the WHO interim guidance, and the definition was very similar to that according to the National Health Commission of the People's Republic of China. Severe disease was defined as respiratory distress or respiratory rate $\geq 30 / \mathrm{min}$, oxygen saturation $\leq 93 \%$ at rest, or the ratio of arterial oxygen partial pressure to fraction of inspired oxygen $\left(\mathrm{PaO}_{2} /\right.$ $\left.\mathrm{FiO}_{2}\right) \leq 300 \mathrm{mmHg}$. Eight studies involving 1,566 patients compared COVID-19 patients having severe disease with those having non-severe disease, including 6 retrospective cohort studies and 2 case series. $14,27,31,36,40,42-44$
Of the 8 studies, 6 studies $(1,111$ patients $)$ compared the proportion of obesity in patients having severe disease with that in patients having non-severe disease, and were of moderate to good quality. ${ }^{14,27,31,36,40,42}$ A metaanalysis found that obesity was significantly associated with more severe disease in hospitalised patients (OR 3.13, 95\% CI 1.41-6.92, P=0.005) (Fig. 3). However, the studies had substantial and significant heterogeneity $\left(\mathrm{Q}=28.8, I^{2}=82.6\right)$, despite 5 of the 6 studies being performed in China. Differences in patient characteristics, investigations and treatment may have contributed to significant variability in these results. The pooled mean BMI was $25.6(24.8-26.4)$ in the severe disease group and $23.0(22.0-24.1)$ in the non-severe disease group of 3 studies. ${ }^{40,43,44}$ The overall mean 


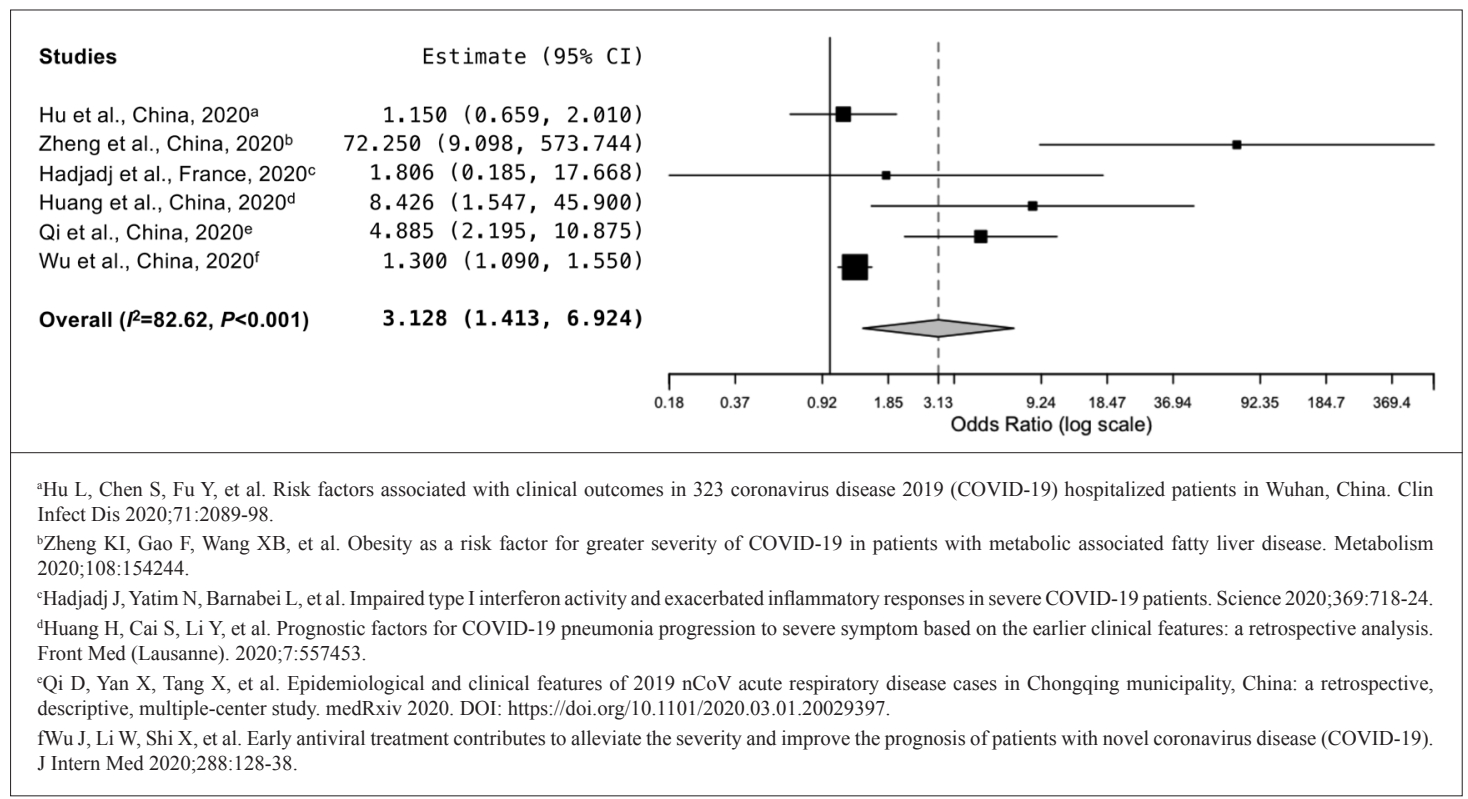

Fig. 3. Meta-analysis of the proportion of COVID-19 patients with obesity, comparing the prevalence in patients with severe disease to those with non-severe disease.

CI: confidence interval; $I^{2}$ : I-squared statistic of heterogeneity

difference in BMI was 2.37 (1.12-3.63, $P<0.001)$, and there was substantial heterogeneity among the studies $\left(\mathrm{Q}=6.9, I^{2}=71.0\right)$. One study combined the severe disease group with the critical disease group. ${ }^{40}$ The sensitivity analysis, which excluded this study by $\mathrm{Wu}$ et al., found that the proportion of obesity remained higher in severe disease (OR 4.89, 95\% CI 1.39-17.18) than that in non-severe disease, with a mean difference in BMI of 3.02 (95\% CI 0.50-5.54).

Notably, the study by Zheng et al. found a particularly large effect size (OR 72.3, 95\% CI 9.1-573.7). ${ }^{42}$ This study only included patients with non-alcoholic fatty liver disease and may not be representative of unselected general hospital patient cohorts used in the other studies. A sensitivity analysis performed excluding this study found no significant effects on the results.

\section{Mortality}

The outcome of mortality was reported in 12 studies involving 45,768 patients, with 11 studies based on hospital inpatients $9,10,12,20,22,25,39,45,46,47,48$ and 1 study in the ICU setting. ${ }^{6}$ Overall, obesity was associated with increased risk of mortality (OR 1.33, 95\% CI 1.07-1.66, $P=0.011)$, with significant and substantial heterogeneity (Q=95.8, $P=88.5)$ (Fig. 4). In the subgroup of studies performed in general hospitalised patients, mortality remained significantly associated with obesity (OR 1.36, 95\% CI 1.09-1.69, $P=0.006$ ) with substantial heterogeneity $\left(\mathrm{Q}=91.7, I^{2}=89.1\right)$. The cohort study performed by Auld et al., ${ }^{6}$ which was the only study investigating the mortality rates in the ICU setting, found a smaller proportion of obese patients in those who died, in contrast to studies performed in the general hospital setting where non-survivors had a larger proportion of obese patients. Further research on obese patients in the ICU setting may be useful in clarifying their prognosis and outcomes in order to investigate the outlier results found in the study by Auld et al.

Peng et al. ${ }^{39}$ showed that obese patients had an OR of 32.1 (95\% CI 6.7-153.0) for mortality that was significantly larger than those in other studies. The study focused on COVID-19 patients with cardiovascular disease first and, within this subpopulation, obese patients were identified, whereas the other studies recruited unselected patients with severe or critical COVID-19. This differing population could explain the higher OR for mortality, given the compounding effect of both cardiovascular disease and obesity on mortality. Exclusion of this study did not have a significant effect on the results of the meta-analysis.

\section{Positive test}

Four studies involving 17,208 patients in the community and hospitals investigated the proportion of obese patients in patients who tested positive for COVID-19 and compared with that in patients who tested negative on RT-PCR of nasopharyngeal swab samples. ${ }^{9,13,19,49}$ The quality of these studies was moderate to good, and 3 


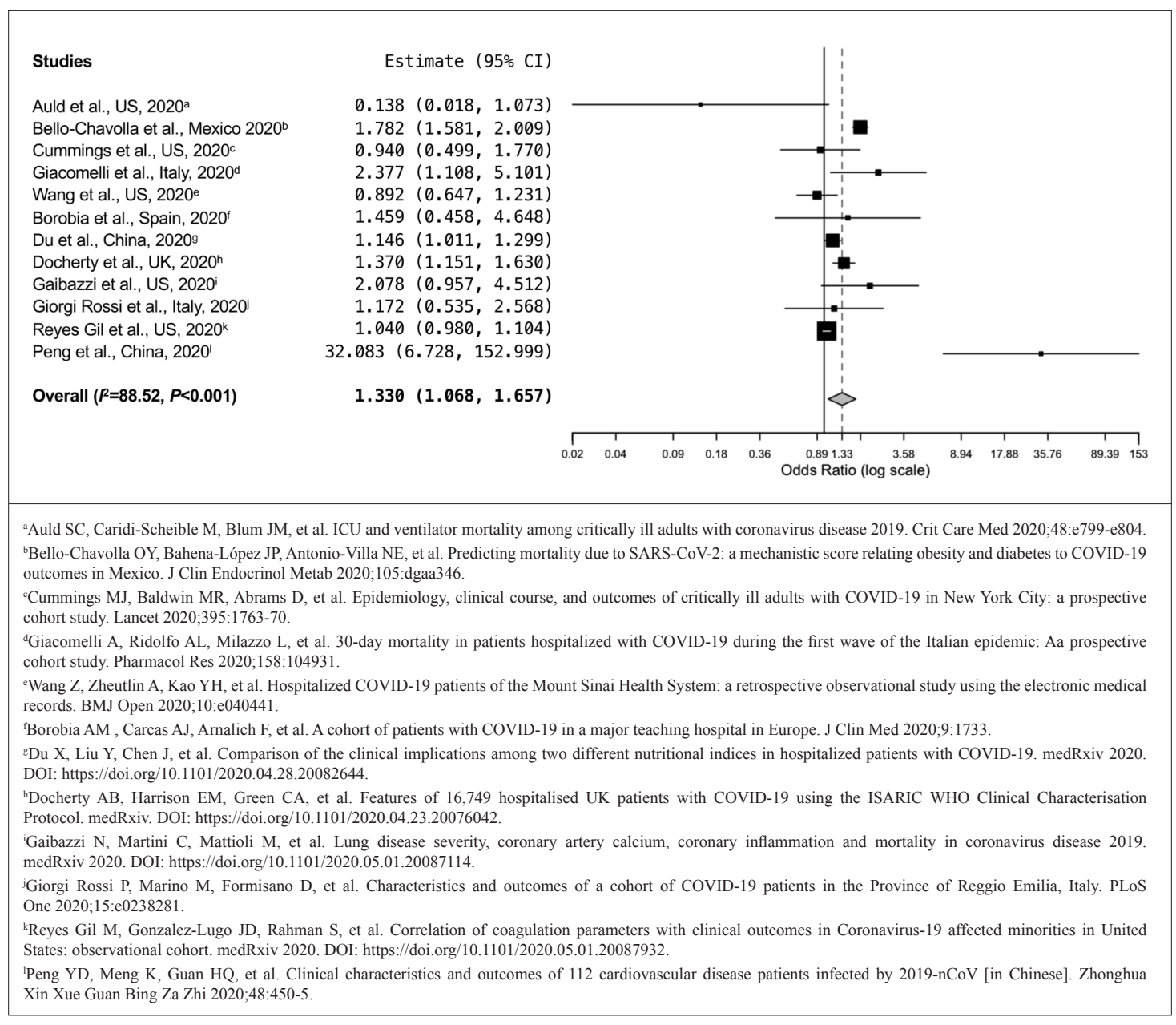

Fig. 4. Meta-analysis of the proportion of COVID-19 patients with obesity, comparing the prevalence in patients with severe disease to those with non-severe disease.

CI: confidence interval; $P$ : I-squared statistic of heterogeneity

were retrospective cohort studies while 1 was a crosssectional study. Meta-analysis found that obesity was significantly associated with a positive COVID-19 test (OR $1.50,95 \%$ CI $1.25-1.81, P<0.001$ ), and there was significant heterogeneity among the studies $(\mathrm{Q}=13.2$, $I^{2}=77.2$; Fig. 5). The study by Vahidy et al ${ }^{19}$ focused on a population from a single health system in the US, in contrast to the use of national databases (e.g. the UK biobank $^{19}$ or the Mexican national database ${ }^{9}$ ) in the other studies in this subgroup analysis.

\section{Discussion}

Obesity and cardiovascular diseases have been linked to viral infections, including influenza, ${ }^{3,50}$ and are risk factors for poor prognosis and severe disease. In this systematic review and meta-analysis, we found that obesity was significantly associated with a positive COVID-19 test, severe disease and mortality, and patients admitted to ICU or with critical disease had significantly higher BMI. Many of the initial observational studies did not report obesity or BMI, and an earlier previous systematic review included 3 studies that concluded that obesity was a risk factor for severe disease and for requirement of advanced medical care. ${ }^{51}$ In this review, we further characterised the prognostic implications of obesity across a larger quantity of subjects and studies compared with previous systematic reviews. ${ }^{52}$

The mechanisms for adverse outcomes in SARSCoV-2 infection associated with obesity may be multifaceted. Mechanically, obesity is associated with reduced diaphragmatic expansion, increased anatomical dead space and increased difficulty of ventilation. Obesity is characterised by a state of chronic low-grade inflammation with elevated levels of proinflammatory cytokines such as interleukin-6, the levels of which were also observed to be elevated in non-survivors compared with survivors in COVID-19. ${ }^{53}$ Leptin 


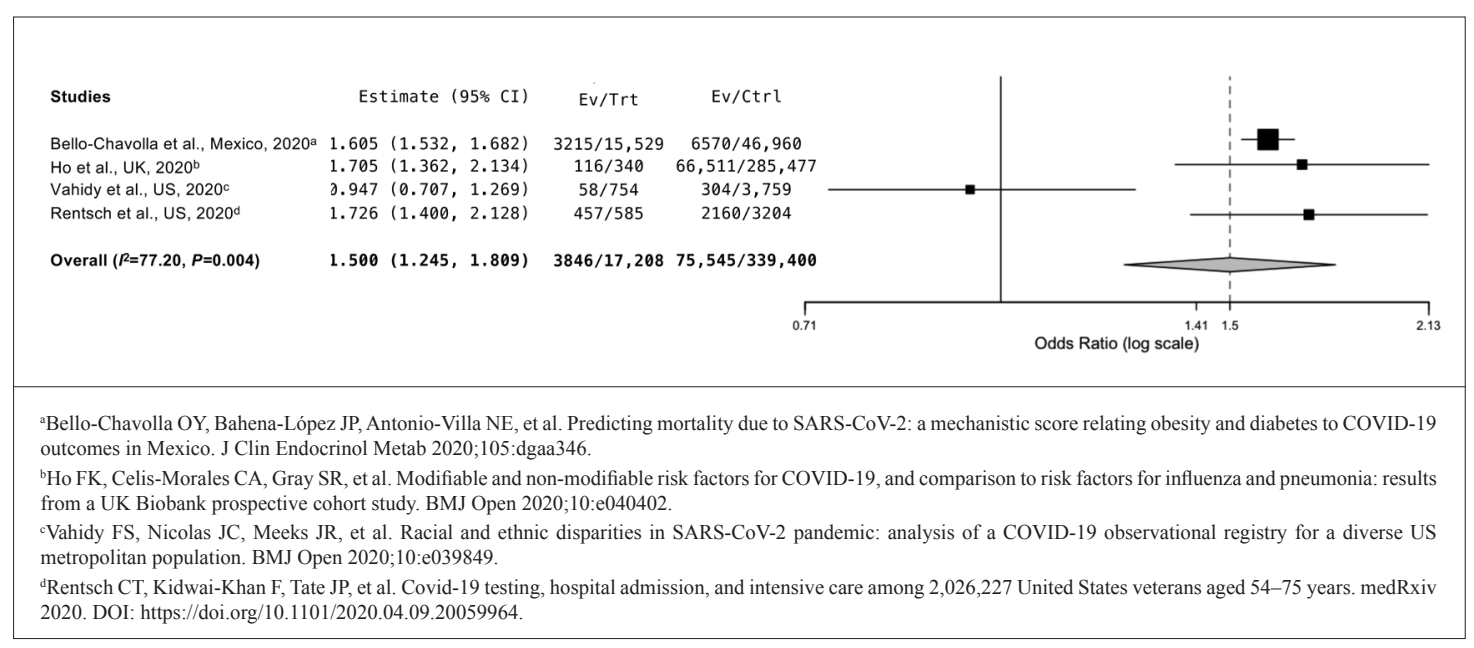

Fig. 5. Meta-analysis of the proportion of COVID-19 patients with obesity, comparing the prevalence in patients who had a positive COVID-19 test to that in patients who tested negative.

CI: confidence interval; $P$ : I-squared statistic of heterogeneity

promotes B cell maturation and inhibits anti-viral $\mathrm{CD} 8^{+} \mathrm{T}$ cell response. Leptin levels are increased in obesity, possibly reducing the effective immune response against viral infections. ${ }^{4}$ Intubated COVID-19 patients were shown to have an elevated leptin level compared with that of non-COVID-19 controls, despite similar BMI, suggesting that leptin may have a role in the pathogenesis of SARS-CoV-2 infection. ${ }^{54}$ Adipose tissue also expresses high levels of angiotensinconverting enzyme- 2 receptors, which mediate entry of SARS-CoV-2.

It is well-known that obesity is associated with diabetes mellitus, coronary artery disease and hypertension, forming the metabolic syndrome. In a cohort of 15,529 COVID-19 patients in Mexico, obese patients were more likely to have hypertension $(33.7 \%$ versus $18.7 \%, P<0.001)$, diabetes $(26.5 \%$ vs $16.2 \%$, $P<0.001)$ and cardiovascular disease $(4.4 \%$ vs $2.6 \%$, $P<0.001) .{ }^{9}$ In multiple meta-analysis of comorbidities in COVID-19, hypertension, diabetes mellitus and cardiovascular disease were found to be associated with severe disease and ICU admission. ${ }^{55}$

Increased BMI was significantly associated with ICU admission or critical illness, and obesity trended towards increasing risk, although not statistically significant. However, results from one study found that the proportion of obese patients who died in ICU was lower than that in survivors. ${ }^{6}$ This should be interpreted with caution owing to lack of data, and the lower mortality rate in obese patients in ICU may be related to the obesity paradox or selection bias of milder obese patients being admitted to ICU. A recent meta-analysis of 6 studies performed by Földi et al. found that patients with obesity had increased risk of ICU admission (OR 1.21, 95\% CI 1.002-1.46), ${ }^{52}$ which, combined with the analysis of 8 studies in this metaanalysis, suggests a relationship between obesity and ICU admission or critical disease.

The studies included in this review were significantly heterogeneous, suggesting that the true effect sizes were different among studies. Zheng et al. focused on a specific population of severe obesity disease (patients with metabolic associated fatty liver disease who are obese as opposed to obese patients alone) which, by definition, will have a compounding effect leading to an increased effect of obesity. ${ }^{42}$ This differing population could explain the outlier result when looking at mortality. The study by Auld et al. ${ }^{7}$ was the only one that specifically focused on an exclusive ITU population, whereas the other studies focused on an unselected general hospital population. Peng et al..$^{39}$ focused on cardiovascular disease patients with COVID-19, whereas most studies focused on unselected patients with severe or critical COVID-19. This differing population could explain the outlier result when looking at mortality. Additionally, cardiovascular comorbidities may confound the effects of obesity on the prognosis of COVID-19. Another cause may be the differences in ethnicity: the prevalence of obesity defined as BMI $\geq 30 \mathrm{~kg} / \mathrm{m}^{2}$ was the lowest in Asians (9.8\%) compared with those of the whites (22.0\%), Latinos (33.6\%) and African Americans (36.1\%) in 42,935 adults in California, US. ${ }^{56}$ Most studies adopted the WHO European definition of obesity, and future research should consider using the Asia-Pacific BMI classification in the Asian population. 
Our meta-analysis also showed that obesity was significantly associated with a positive COVID-19 test (OR $1.50,95 \%$ CI $1.25-1.81, P<0.001$ ). This finding adds to the evidence that obesity leads to increased infectivity of COVID-19 (positive tests as a proxy for infectivity). Most studies reported testing on symptomatic patients, which was the widely adopted method of testing during this stage of the pandemic, and it would be interesting to see studies of asymptomatic patients. ${ }^{57}$ Recent large national studies utilising the UK primary care network ${ }^{58}$ and the Information System for Research in Primary Care in Spain ${ }^{59}$ found that obesity was associated with increased risk of positive COVID-19 testing. Reporting of obesity and positive test results may be a measure of how susceptible obese patients are to catching the virus, which could have implications on public health policies and healthcare utilisation.

Limitations of this study include the possibility of confounding factors and heterogeneity of the included studies. The heterogeneity in studies, mainly concentrating on study design, populations studied, geographical areas and definitions of COVID-19 severity used, will inevitably make it harder to draw definitive conclusions about the relationship between obesity and COVID-19 from across the literature. Only a subgroup of studies reported each of our identified primary and secondary outcomes, increasing the risk of type 1 and type 2 error in our conclusions. Seventeen articles from pre-print servers such as medRxiv that were not peer-reviewed but were included to ensure that the literature search was comprehensive, and the quality of these studies were assessed using validated tools. Publication bias was not assessed owing to the small number of studies in each meta-analysis.

\section{Conclusion}

Obesity was associated with an increased risk of severe disease, mortality and positive test for SARS$\mathrm{CoV}-2$ infection. Obesity trends towards higher risk of ICU admission and critical illness, and a higher BMI, rather than obesity, is associated with a statistically significant increase in risk of ICU admission and critical illness. Overall, this study suggests that obesity may be a marker of poor prognosis, and with the additional mechanistic challenges in ventilation, obese patients should be monitored closely and managed carefully. Compared with recently published systematic reviews and meta-analyses ${ }^{51,52}$ we analysed several important clinical outcomes (critical disease, severe disease and mortality) and comprehensively searched the literature including pre-print texts and clinical trials for evidence, increasing the sample of studies from which to draw conclusions. Our findings support previous findings in the other systematic reviews. Future studies on COVID-19 patients should report prevalence of obesity and BMI in order to increase the understanding of the interaction between obesity and SARS-CoV-2 infection, and to inform the clinical management of these patients.

\section{REFERENCES}

1. Chew SY, Lee YS, Ghimiray D, et al. Characteristics and outcomes of COVID-19 patients with respiratory failure admitted to a "pandemic ready" intensive care unit - lessons from Singapore. Ann Acad Med Singap 2020;49:434-48.

2. WHO Expert Consultation. Appropriate body-mass index for Asian populations and its implications for policy and intervention strategies. Lancet 2004;363:157-63.

3. Morgan OW, Bramley A, Fowlkes A, et al. Morbid obesity as a risk factor for hospitalization and death due to 2009 pandemic influenza A(H1N1) disease. PLoS One 2010;5:e9694.

4. Michalakis K, Ilias I. SARS-CoV -2 infection and obesity: common inflammatory and metabolic aspects. Diabetes Metab Syndr 2020;14:469-71.

5. Caussy C, Pattou F, Wallet F, et al. Prevalence of obesity among adult inpatients with COVID-19 in France. Lancet Diabetes Endocrinol 2020;8:562-4.

6. Auld SC, Caridi-Scheible M, Blum JM, et al. ICU and ventilator mortality among critically ill adults with coronavirus disease 2019. Crit Care Med 2020;48:e799-e804

7. Argenziano MG, Bruce SL, Slater CL, et al. Characterization and clinical course of 1000 patients with coronavirus disease 2019 in New York: retrospective case series. BMJ 2020;369:m1996.

8. Barrasa H, Rello J, Tejada S, et al. SARS-CoV-2 in Spanish intensive care units: early experience with 15-day survival in Vitoria. Anaesth Crit Care Pain Med 2020;39:553-61.

9. Bello-Chavolla OY, Bahena-López JP, Antonio-Villa NE, et al. Predicting mortality due to SARS-CoV-2: a mechanistic score relating obesity and diabetes to COVID-19 outcomes in Mexico. J Clin Endocrinol Metab 2020;105:dgaa346.

10. Cummings MJ, Baldwin MR, Abrams D, et al. Epidemiology, clinical course, and outcomes of critically ill adults with COVID-19 in New York City: a prospective cohort study. Lancet 2020;395:1763-70.

11. Ebinger JE, Achamallah N, Ji H, et al. Pre-existing traits associated with COVID-19 illness severity. PLoS One 2020;15:e0236240.

12. Giacomelli A, Ridolfo AL, Milazzo L, et al. 30-day mortality in patients hospitalized with COVID-19 during the first wave of the Italian epidemic: a prospective cohort study. Pharmacol Res 2020;158:104931.

13. Ho FK, Celis-Morales CA, Gray SR, et al. Modifiable and nonmodifiable risk factors for COVID-19, and comparison to risk factors for influenza and pneumonia: results from a UK Biobank prospective cohort study. BMJ Open 2020;10:e040402.

14. $\mathrm{Hu} \mathrm{L}, \mathrm{Chen} \mathrm{S}, \mathrm{Fu} \mathrm{Y}$, et al. Risk factors associated with clinical outcomes in 323 coronavirus disease 2019 (COVID-19) hospitalized patients in Wuhan, China. Clin Infect Dis 2020;71:2089-98.

15. Kalligeros M, Shehadeh F, Mylona EK, et al. Association of obesity with disease severity among patients with coronavirus disease 2019 . Obesity (Silver Spring) 2020;28:1200-4. 
16. Petrilli CM, Jones SA, Yang J, et al. Factors associated with hospital admission and critical illness among 5279 people with coronavirus disease 2019 in New York City: prospective cohort study. BMJ 2020;369:m1966.

17. Piva S, Filippini M, Turla F, et al. Clinical presentation and initial management critically ill patients with severe acute respiratory syndrome coronavirus 2 (SARS-CoV-2) infection in Brescia, Italy. J Crit Care 2020;58:29-33.

18. Simonnet A, Chetboun M, Poissy J, et al. High prevalence of obesity in severe acute respiratory syndrome coronavirus-2 (SARS-CoV-2) requiring invasive mechanical ventilation. Obesity (Silver Spring) 2020;28:1195-9.

19. Vahidy FS, Nicolas JC, Meeks JR, et al. Racial and ethnic disparities in SARS-CoV-2 pandemic: analysis of a COVID-19 observational registry for a diverse US metropolitan population. BMJ Open 2020;10:e039849.

20. Borobia AM, Carcas AJ, Arnalich F, et al. A cohort of patients with COVID-19 in a major teaching hospital in Europe. JClin Med 2020;9:1733.

21. Caussy C, Wallet F, Laville M, et al. Obesity is associated with severe forms of COVID-19. Obesity (Silver Spring) 2020;28:1175.

22. Gaibazzi N, Martini C, Mattioli M, et al. Lung disease severity, coronary artery calcium, coronary inflammation and mortality in coronavirus disease 2019. medRxiv 2020. DOI: https://doi.org/10.110 $1 / 2020.05 .01 .20087114$

23. Garg S, Kim L, Whitaker M, et al. Hospitalization rates and characteristics of patients hospitalized with laboratory-confirmed coronavirus disease 2019 - COVID-NET, 14 states, March 1-30, 2020. MMWR Morb Mortal Wkly Rep 2020;69:458-64

24. Rodríguez-Cola M, Jiménez-Velasco I, Gutiérrez-Henares F, et al. Clinical features of coronavirus disease 2019 (COVID-19) in a cohort of patients with disability due to spinal cord injury. Spinal Cord Ser Cases 2020;6:39.

25. Giorgi Rossi P, Marino M, Formisano D, et al. Characteristics and outcomes of a cohort of COVID-19 patients in the Province of Reggio Emilia, Italy. PLoS One 2020;15:e0238281.

26. Guo AX, Cui JJ, OuYang QY, et al. The clinical characteristics and mortal causes analysis of COVID-19 death patients. medRxiv 2020. DOI: https://doi.org/10.1101/2020.04.12.20062380.

27. Huang H, Cai S, Li Y, et al. Prognostic factors for COVID-19 pneumonia progression to severe symptom based on the earlier clinical features: a retrospective analysis. Front Med (Lausanne) 2020;7:557453.

28. Lighter J, Phillips M, Hochman S, et al. Obesity in patients younger than 60 years is a risk factor for COVID-19 hospital admission. Clin Infect Dis 2020;71:896-7.

29. Mahévas M, Tran V-T, Roumier M et al. Clinical efficacy of hydroxychloroquine in patients with covid-19 pneumonia who require oxygen: observational comparative study using routine care data. BMJ 2020;369:m1844.

30. Menter T, Haslbauer JD, Nienhold R, et al. Post-mortem examination of COVID-19 patients reveals diffuse alveolar damage with severe capillary congestion and variegated findings of lungs and other organs suggesting vascular dysfunction. Histopathology 2020;77:198-209.

31. Qi D, Yan X, Tang X, et al. Epidemiological and clinical features of 2019-nCoV acute respiratory disease cases in Chongqing municipality, China: a retrospective, descriptive, multiple-center study. medRxiv 2020. DOI: https://doi.org/10.1101/2020.03.01.20029397.

32. Richardson S, Hirsch JS, Narasimhan M, et al. Presenting characteristics, comorbidities, and outcomes among 5700 patients hospitalized with COVID-19 in the New York City area. JAMA 2020;323:2052-9

33. Vuagnat P, Frelaut M, Ramtohul T, et al. COVID-19 in breast cancer patients: a cohort at the Institut Curie hospitals in the Paris area. Breast Cancer Res 2020;22:55.

34. Wollenstein-Betech S, Cassandras CG, Paschalidis IC. Personalized predictive models for symptomatic COVID-19 patients using basic preconditions: hospitalizations, mortality, and the need for an ICU or ventilator. Int J Med Inform 2020;142:104258.

35. Zuo Y, Yalavarthi S, Shi H, et al. Neutrophil extracellular traps (NETs) as markers of disease severity in COVID-19. medRxiv 2020. DOI: https://doi.org/10.1101/2020.04.09.20059626.

36. Hadjadj J, Yatim N, Barnabei L, et al. Impaired type I interferon activity and exacerbated inflammatory responses in severe COVID-19 patients. Science 2020;369:718-24.

37. Lau FH, Majumder R, Torabi R, et al. Vitamin D insufficiency is prevalent in severe COVID-19. medRxiv 2020. DOI: https://doi.org/10 .1101/2020.04.24.20075838.

38. Valente-Acosta B, Hoyo-Ulloa I, Espinosa-Aguilar L, et al. COVID-19 severe pneumonia in Mexico City - first experience in a Mexican hospital. medRxiv 2020. DOI: https://doi.org/10.1101/202 0.04.26.20080796.

39. Peng YD, Meng K, Guan HQ, et al. Clinical characteristics and outcomes of 112 cardiovascular disease patients infected by 2019-nCoV [in Chinese]. Zhonghua Xin Xue Guan Bing Za Zhi 2020;48:450-5.

40. Wu J, Li W, Shi X, et al. Early antiviral treatment contributes to alleviate the severity and improve the prognosis of patients with novel coronavirus disease (COVID-19). J Intern Med 2020;288:128-38.

41. Abou-Arab O, Huette P, Berna P, et al. Tracheal trauma after difficult airway management in morbidly obese patients with COVID-19. Br J Anaesth 2020;125:e168-e170.

42. Zheng KI, Gao F, Wang XB, et al. Obesity as a risk factor for greater severity of COVID-19 in patients with metabolic associated fatty liver disease. Metabolism 2020;108:154244

43. Chen Q, Zheng Z, Zhang C, et al. Clinical characteristics of 145 patients with corona virus disease 2019 (COVID-19) in Taizhou, Zhejiang, China. Infection 2020;48:543-51

44. Liu M, He P, Liu HG, et al. Clinical characteristics of 30 medical workers infected with new coronavirus pneumonia [in Chinese]. Zhonghua Jie He He Hu Xi Za Zhi 2020;43:209-14.

45. Wang Z, Zheutlin A, Kao YH, et al. Hospitalized COVID-19 patients of the Mount Sinai Health System: a retrospective observational study using the electronic medical records. BMJ Open 2020; 10:e040441.

46. Du X, Liu Y, Chen J, et al. Comparison of the clinical implications among two different nutritional indices in hospitalized patients with COVID-19. medRxiv 2020. DOI: https://doi.org/10.1101/2020.0 4.28.20082644.

47. Docherty AB, Harrison EM, Green CA, et al. Features of 16,749 hospitalised UK patients with COVID-19 using the ISARIC WHO Clinical Characterisation Protocol. medRxiv 2020. DOI: https://doi.org 10.1101/2020.04.23.20076042.

48. Reyes Gil M, Gonzalez-Lugo JD, Rahman S, et al. Correlation of coagulation parameters with clinical outcomes in Coronavirus-19 affected minorities in United States: observational cohort. medRxiv 2020. DOI: https://doi.org/10.1101/2020.05.01.20087932.

49. Rentsch CT, Kidwai-Khan F, Tate JP, et al. Covid-19 testing, hospital admission, and intensive care among 2,026,227 United 
States veterans aged 54-75 years. medRxiv 2020. DOI: https://doi.org/ 10.1101/2020.04.09.20059964

50. Ho JSY, Tambyah PA, Sia CH. A call for vaccine against COVID-19: Implications for cardiovascular morbidity and healthcare utilization. Cardiovasc Drugs Ther 2020;34:585-7.

51. Tamara A, Tahapary DL. Obesity as a predictor for a poor prognosis of COVID-19: a systematic review. Diabetes Metab Syndr 2020; 14:655-9.

52. Földi M, Farkas N, Kiss S, et al. Obesity is a risk factor for developing critical condition in COVID-19 patients: A systematic review and meta-analysis. Obes Rev 2020;21:e13095.

53. Ruan Q, Yang K, Wang W, et al. Clinical predictors of mortality due to COVID-19 based on an analysis of data of 150 patients from Wuhan, China. Intensive Care Med 2020;46:846-8.

54. van der Voort PHJ, Moser J, Zandstra DF, et al. Leptin levels in SARS-CoV-2 infection related respiratory failure: A cross-sectional study and a pathophysiological framework on the role of fat tissue. Heliyon 2020;6:e04696.
55. Ho JS, Tambyah PA, Ho AF, et al. Effect of coronavirus infection on the human heart: A scoping review. Eur J Prev Cardiol 2020; 27:1136-48.

56. Wang L, Southerland J, Wang K, et al. Ethnic differences in risk factors for obesity among adults in California, the United States. J Obes 2017;2017:2427483.

57. Zhou JC, Hui TC, Tan $\mathrm{CH}$, et al. Chest radiography in coronavirus disease 2019 (COVID-19): Correlation with clinical course. Ann Acad Med Singap 2020;49:456-61.

58. de Lusignan S, Dorward J, Correa A, et al. Risk factors for SARSCoV-2 among patients in the Oxford Royal College of General Practitioners Research and Surveillance Centre primary care network: a cross-sectional study. Lancet Infect Dis 2020;20:1034-42.

59. Burn E, Tebe C, Fernandez-Bertolin S, et al. The natural history of symptomatic COVID-19 in Catalonia, Spain: A multi-state model including 109,367 outpatient diagnoses, 18,019 hospitalisations, and 5,585 COVID-19 deaths among 5,627,520 people. medRxiv 2020. DOI: https://doi.org/10.1101/2020.07.13.20152454 